\title{
Short Bowel Syndrome from Strangulated Internal Hernia After Childbirth in a Patient With a History of Gastric Bypass Surgery: Case Report
}

\author{
Jisu Lee a, Tae Sun Ha a,b,c,*, Jeonghwi Choi c,d, Yeonhwa Kwon c,e, Zisun Kim a \\ a Department of Surgery, Soonchunhyang University College of Medicine, Bucheon Hospital, Bucheon, Korea \\ ${ }^{b}$ Subdivision of Critical Care Medicine, Soonchunhyang University College of Medicine, Bucheon Hospital, Bucheon, Korea \\ ${ }^{c}$ Nutritional Support Team, Soonchunhyang University College of Medicine, Bucheon Hospital, Bucheon, Korea \\ ${ }^{d}$ Department of Pharmacy, Soonchunhyang University College of Medicine, Bucheon Hospital, Bucheon, Korea \\ ${ }^{e}$ Department of Food and Nutrition, Soonchunhyang University College of Medicine, Bucheon Hospital, Bucheon, Korea
}

\section{Article history:}

Received: August 6, 2019

Revised: September 2, 2019

Accepted: January 29, 2020

\section{*Corresponding Author:}

\section{Tae Sun Ha}

Department of Surgery, Soonchunyang

University Bucheon Hospital, Bucheon, 170

Jomaru-ro, Bucheon, Gyeonggi-do, 14584,

Korea

E-mail: applips@gmail.com

\section{ORCID}

Jisu Lee

https://orcid.org/0000-0003-4971-5069

Tae Sun $\mathrm{Ha}$

https://orcid.org/0000-0003-3683-6929

Jeonghwi Choi

https://orcid.org/0000-0003-1183-6631

YeonHwa Kwon

https://orcid.org/0000-0002-9090-0787

Zisum Kim

https://orcid.org/0000-0002-1413-2800

\section{Introduction}

Bariatric surgery has proven to be the most effective and efficient therapeutic option for achieving sustainable and significant weight loss. However, bariatric patients are at risk of a variety of complications, such as bowel obstruction, internal hernia, and mesenteric thrombosis, which can result in massive small bowel resection, with short bowel syndrome (SBS) as a consequence [1]. The majority of patients who undergo bariatric surgery are females of child bearing-age, and complications associated with such surgery have been reported in subsequent pregnancies [2]. Here, a case of extensive short bowel syndrome resulting from massive small bowel resection of an internal hernia after childbirth in a patient with a history of gastric bypass surgery is reported.

\section{Case Report}

A 36-year-old female (gravida 2, para 0) presented to the Emergency Department with drowsiness and dyspnea the day after a cesarean section at a local hospital. She had undergone a laparoscopic Roux-en-Y gastric bypass (RYGB) 3 years earlier and had lost $25 \mathrm{~kg}$ before becoming pregnant. Upon arrival, 
the patient's blood pressure was not measured, but her heart rate was 151 beats/minute, respiratory rate was 28 breaths/ minute, and body temperature was $37.0^{\circ} \mathrm{C}$. Blood oxygen saturation (SpO2) was $88 \%$ following $02(10 \mathrm{~L})$ inhalation via the Back-Valve-Mask Ambu bag. Fluid resuscitation was started with vasopressors (norepinephrine $0.3 \mu \mathrm{g} / \mathrm{kg} /$ minute) and mechanical ventilation was implemented after intubation. Due to the patient's drowsiness, a limited physical examination was performed, but coarse breathing sounds were noted on both lungs and a distended abdomen without muscle guarding was detected. Arterial blood gas analysis revealed that the patient had severe metabolic acidosis (pH 6.99, $\mathrm{PCO} 251 \mathrm{mmHg}, \mathrm{PaO} 2$ 50mmHg, HCO3 $11.8 \mathrm{mmol} / \mathrm{L}, \mathrm{BE}-19.5 \mathrm{mmol} / \mathrm{L}$, lactic acid 2.74 $\mathrm{mmol} / \mathrm{L}$ ). The laboratory data were as follows: hemoglobin 8.6 $\mathrm{g} / \mathrm{dL}$, white blood cell count $18,760 / \mu \mathrm{L}$ (segment neutrophils $65 \%$ ), platelet count 192,000 , blood urea nitrogen $30.9 \mathrm{mg} /$ $\mathrm{dL}$, creatinine $2.5 \mathrm{mg} / \mathrm{dL}$, total bilirubin $0.47 \mathrm{mg} / \mathrm{dL}$, aspartate aminotransferase $23 \mathrm{IU} / \mathrm{L}$, alanine aminotransferase $10 \mathrm{IU} /$ $\mathrm{L}$, and high-sensitivity C-reactive protein $5.59 \mathrm{mg} / \mathrm{dL}$. The coagulation profile was normal. Based on a focused assessment with sonography for trauma which had positive findings on 3 views, she received an emergency laparotomy because of a suspicion of intra-abdominal bleeding due to uterine rupture or bleeding at the operation site. An emergency laparotomy revealed a large (4-5 L) ascites collection, without uterine rupture or intra-abdominal bleeding. Bowel conditions such as bowel color, distension, or internal herniation could not be carefully checked due to the previous Pfannenstiel incision from the first operation. The patient was admitted to the Department of Obstetrics and Gynecology and was treated in the surgical intensive care unit to rule out the possibility of an amniotic fluid embolism. On Day 1 of hospitalization, the patient had acute abdominal pain with hematemesis, and she received an emergency esophagogastroduodenoscopy. Small bowel ischemia with mucosal bleeding was identified at

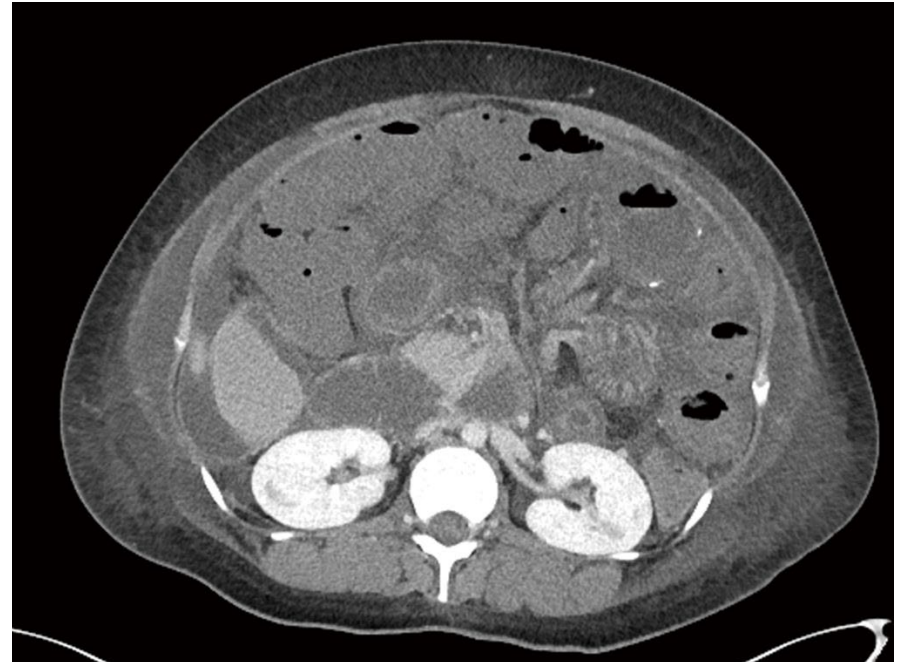

Figure 2. Abdominopelvic computed tomography scan showed strangulated obstruction of the small bowel with extensive ischemic change and mesenteric congestion.

the anastomotic site of the RYGB (Figure 1). She experienced progressive hemodynamic instability and her blood pressure dropped to $61 / 49 \mathrm{mmHg}$. Her heart rate was $140-150$ beats/ minute, respiratory rate was $25-35$ breaths/minute, and body temperature was above $38.0^{\circ} \mathrm{C}$. She received aggressive fluid therapy with high-dose vasopressors (norepinephrine 0.5 $\mu \mathrm{g} / \mathrm{kg} /$ minute, vasopressin $0.03 \mathrm{mg} /$ minute $/ \mathrm{kg}$ ) and broadspectrum antibiotics (vancomycin and meropenem). Abdominal computed tomography (CT) scans with contrast showed an obstruction of the small bowel with extensive small bowel ischemia and congestion (Figure 2). The Department of General Surgery were contacted to perform an emergency laparotomy. It revealed an internal hernia in Petersen's space with volvulus, causing extensive small bowel infarction and necrosis. After small bowel resection and double barrel enterostomy, the lengths of residual small intestine were distal $50 \mathrm{~cm}$ from

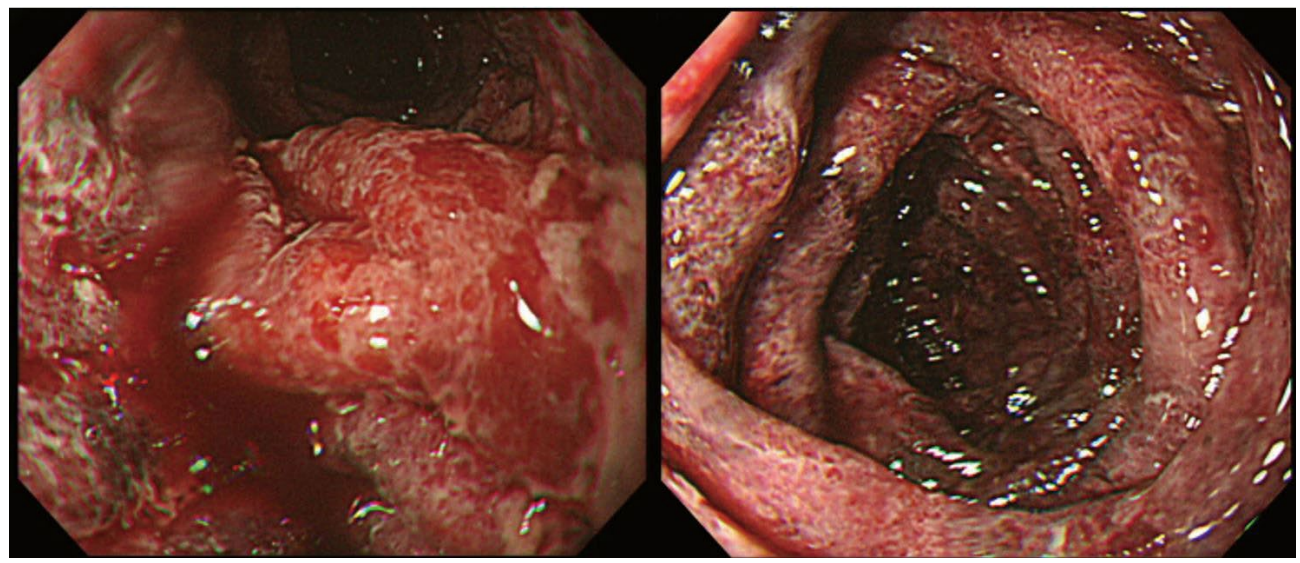

Figure 1. Diffuse small bowel ischemia and necrosis with mucosal bleeding was observed during an esophagogastroduodenoscopy. 
(A)

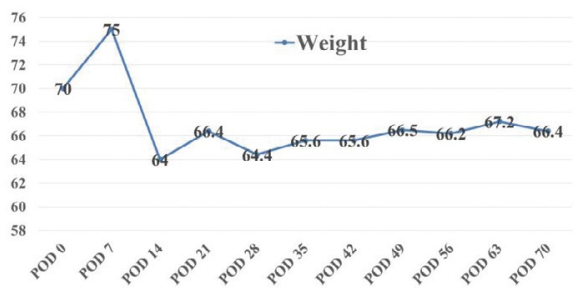

(D)

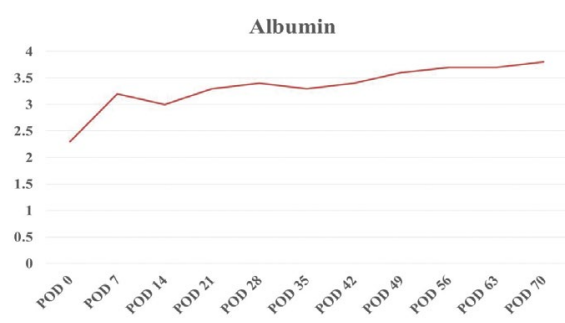

(B)

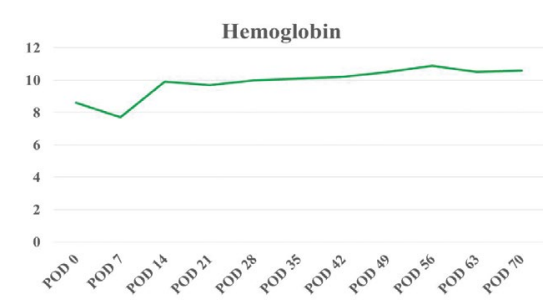

(E)

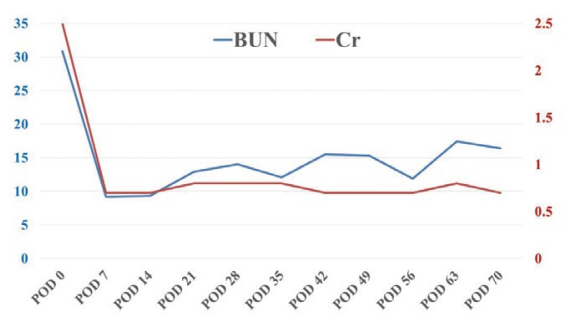

(C)

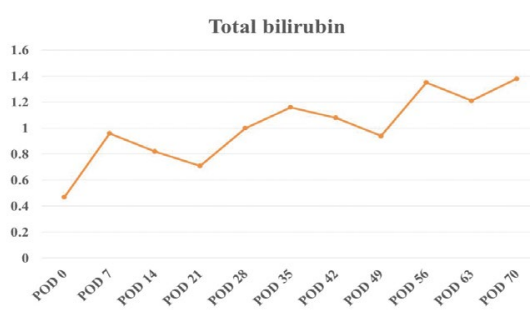

(F)

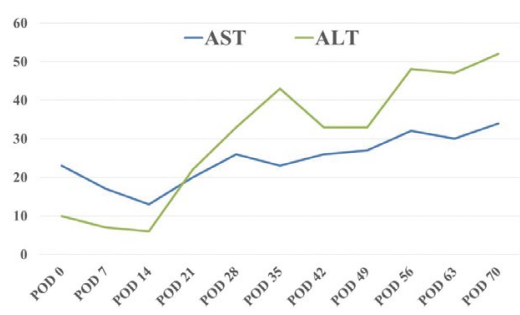

Figure 3. Biochemical follow up results during hospitalization. (A) Weight, (B) Hemoglobin, (C) Total bilirubin, (D) Albumin, (E) BUN and Creatinine, (F) AST/ALT.

ALT = alanine aminotransferase; AST = aspartate aminotransferase; BUN = blood urea nitrogen; POD = postoperative day.

the Treitz ligament, and proximal $30 \mathrm{~cm}$ from the ileocecal valve. She received supportive care and nutritional support for extensive short bowel syndrome from a multidisciplinary team, consisting of an intensivist, a pharmacist, and a dietitian. The patient was transferred to a general ward on the 9 th postoperative day. She received fluid replacement and commercial parenteral nutrition (PN) for prevention of dehydration and malnutrition during her hospital stay because of the large amount of fluid loss via the jejunostomy (2-4 L/ day). Due to excessive gastrointestinal fluid and failure of adaptation, the patient could not be switched from PN to oral/ enteral feeding alone. She was managed with PN supplemented with electrolytes, vitamins, and trace elements, based on a nutritional assessment. During a treatment period of 74 days, the patient did not suffer significant weight loss, nutrient deficiency, electrolyte imbalance, or adverse effect of PN (Figure 3). She was referred to the Transplant Center for possible small bowel transplantation.

\section{Discussion}

In recent years, gastric bypass surgery which creates a very small functional stomach for reducing food intake has become one of the most popular operations and has provided the best long-term outcomes [3]. The safety of pregnancy after RYGB has been well documented, but women undergoing this surgery are still at risk for anastomic leaks, gastrointestinal bleeding, small intestine obstruction, and internal herniation [4]. Among these complications, the most common, and sometimes lifethreatening, is internal herniation of the bowel. Laparoscopic RYGB results in fewer postoperative adhesions, and thus reduces fixation of the small bowel to the abdominal wall, leading to increased risk of internal hernia of the bowel loop [5]. It has been observed that the majority of these internal hernias present months or years, not days after surgery. Internal hernia has been shown to develop when weight loss causes a rapid decrease in mesenteric fat, enlarging the surgically-created mesenteric defects and causing the loosening of mesenteric sutures. Potential internal hernia locations were the transverse mesocolon defect, jejunostomy mesenteric defect, and the space between the transverse mesocolon and the Roux-en-Y limb mesentery (Petersen's space) [6]. Pregnancy specifically increases the risk of formation of an internal herniation because of the increased intra-abdominal pressure and displacement of intra-abdominal contents by a gravid uterus, which can increase the possibility of the bowel herniating through mesenteric defect [7]. The patient presented several years after the laparoscopic RYGB, after she had lost almost 30 $\mathrm{kg}$. It is speculated that an internal hernia within Petersen's space developed with volvulus after rapid involution of the postpartum uterus.

An internal hernia is a recognized complication after a RYGB, with an incidence ranging from less than $1 \%$ up to $16 \%$ [8]. Internal hernias can cause intestinal obstruction and volvulus, leading to ischemia and/or gangrene, and anastomotic 
disruption, as well as dilatation of the bypassed stomach. Patients present with a spectrum of nonspecific signs and symptoms ranging from mild intermittent abdominal pain, to vomiting. Late signs and symptoms when left untreated included peritonitis, sepsis, metabolic acidosis, and shock. Pregnant women with a history of RYGB who present with abdominal pain present a unique challenge for diagnosis. Abdominal pain, as well as vomiting are common complaints in pregnancy, and differentiating this from an internal hernia can be difficult. Leal-Gonzalez et al summarized 15 articles studying internal hernias in pregnant women with a history of RYGB [4]. The most common symptoms were abdominal pain ( $n=22,100 \%)$, nausea ( $n=12,54.5 \%$ ), vomiting ( $n=10$, $45.5 \%)$, and uterine contraction $(n=1,4.5 \%)$, and 2 patients (9\%) experienced severe abdominal pain. Most patients waited at least 2 days (range from 30 minutes to 6 weeks) before consulting with their physician. Both intraperitoneal and subcutaneous fat minimize tenderness and mask peritoneal signs. Furthermore, initial vital signs and laboratory results were reported to be within the normal range in most cases. Use of abdominal CT may be limited in pregnant patients due to their concerns about radiation exposure to the fetus, although abdomen CT scans rarely exceed $25 \mathrm{mGy}$, and have an almost negligible effect on the fetus below doses of $50 \mathrm{mGy}$ [7]. Initially, minimal abdominal distension and lack of parietal involvement can underestimate the severity of disease and lead to misdiagnosis. If peritonitis findings are present, such as rebound tenderness and muscle guarding upon physical examination, transmural necrosis may have occurred, and the prognosis would be poor. The only way to avoid diagnosis delay and devastating bowel necrosis, is to be acutely aware of the potential for internal hernias in women with a history of bariatric surgery and subsequent pregnancy. In patients with intermittent abdominal pain and emesis after a RYGB, surgical consultation should not be delayed, regardless of the patient's apparent condition. Surgical exploration is recommended if internal herniation is suspected, even if laboratory results, and physical examination are unremarkable, and radiological studies are negative [1,9]. The patients past history of undergoing a RYGB in the present case was overlooked, and the diagnosis was missed during the first exploration. A high level of suspicion of herniation should always be present, especially if a laparotomy is performed under an unexplained diagnosis. The ways to reduce the incidence of an internal hernia during pregnancy include postponing pregnancy for least 18 months after gastric bypass surgery, close both mesenteric and Petersen's space defects, use non-absorbable interrupted sutures for closing the mesenteric defect, and to suture without excessive tension at the gastrojejunal and jejunostomy anastomosis [9]. Patients with SBS have severe diarrhea, secondary to failure to absorb gastrointestinal secretions, resulting in dehydration, and an electrolyte imbalance. The incidence of protein-calorie malnutrition with severe hypoalbuminemia was reported to be remarkable, and micronutrient deficiencies, including lipid soluble vitamins, iron, zinc, and magnesium, were commonly observed in patients with SBS [10]. In this current study, PN was initiated on the first postoperative day to prevent severe weight loss, according to the long-term nutritional support strategy of the multidisciplinary nutrition team. The patient rapidly recovered without complications of PN.

The limitation in this case was that the physicians did not consider an internal hernia diagnosis in a patient who had a gastric bypass at an Emergency Department previously, due to lack of clinical experience.

In conclusion, an internal hernia with volvulus caused SBS due to extensive small bowel resection in a patient with a history of a laparoscopic RYGB. The possibility of an internal hernia should always be considered in pregnant, postbariatric surgery patients who present with abdominal pain. Even if vital signs, laboratory results, and imaging studies are normal, it is important to have an acute awareness of potential herniation and without delay to perform a surgical exploration, in an effort to avoid devastating bowel strangulation and sepsis, culminating in the loss of both fetus and mother. SBS is complicated and multifaceted requiring a multidisciplinary approach including medical, nutritional, and pharmaceutical therapies, to optimize fluid and nutrient absorption.

\section{Conflicts of Interest}

The authors have no conflicts of interest relevant to this article.

\section{Acknowledgements}

This research was supported by Soonchunhyang University Research.

\section{References}

[1] McBride CL, Petersen A, Sudan D, Thompson J. Short bowel syndrome following bariatric surgical procedures. Am J Surg 2006;192(6):828-32.

[2] Moore KA, Ouyang DW, Whang EE. Maternal and fetal deaths after gastric bypass surgery for morbid obesity. N Engl J Med 2004;351(7):721-2.

[3] Fried M, Yumuk V, Oppert JM, Scopinaro N, Torres A, Weiner R, et al. Interdisciplinary European guidelines on metabolic and bariatric surgery. Rozhl Chir 2014;93(7):366-78. [in Czech]

[4] Leal-Gonzalez R, De la Garza-Ramos R, Guajardo-Perez H, Ayala-Aguilera $\mathrm{F}$, Rumbaut R. Internal hernias in pregnant women with history of gastric bypass surgery: Case series and review of literature. Int J Surg Case Rep 2013;4(1):44-7.

[5] Ahmed AR, O'Malley W. Internal hernia with Roux loop obstruction during pregnancy after gastric bypass surgery. Obes Surg 2006;16(9):1246-8. 
[6] Schweitzer MA, DeMaria EJ, Broderick TJ, Sugerman HJ. Laparoscopic closure of mesenteric defects after Roux-en-Y gastric bypass. J Laparoendosc Adv Surg Tech A 2000;10(3):173-5.

[7] Bebber FE, Rizzolli J, Casagrande DS, Rodrigues MT, Padoin AV, Mottin CC, et al. Pregnancy after bariatric surgery: 39 pregnancies follow-up in a multidisciplinary team. Obes Surg 2011;21(10):1546-51.

[8] Scheirey CD, Scholz FJ, Shah PC, Brams DM, Wong BB, Pedrosa M Radiology of the laparoscopic Roux-en-Y gastric bypass procedure: Conceptualization and precise interpretation of results. Radiographics 2006;26(5):1355-71.
[9] Morgan H, Chastanet R, Lucha PA Jr. Internal hernia after laparoscopic gastric bypass surgery: A case report and literature review. Postgrad Med 2008;120(2):E01-5.

[10] Stein J, Stier C, Raab H, Weiner R. Review article: The nutritional and pharmacological consequences of obesity surgery. Aliment Pharmacol Ther 2014;40(6):582-609. 